\title{
A Study on the Model For Mesoscopic Heterogenous Rocks Under Uniaxial Tensile Loading
}

\author{
Yunhua Zhu \\ School of Engineering and Technology, Neijiang Normal University, Neijiang 641112,China \\ zhuyh75@126.com
}

Keywords: uniaxial tensile loading; mesoscopic heterogenous rock; damage mechanics Abstract: Stress redistribution induced by excavation often results in tensile zones in the surrounding rock mass. It is of great theoretical and practical significances to analyse the localizations of deformation and damage and to study the complete stress-strain relation for mesoscopic heterogenous rocks under uniaxial tensile loading. Based on damage mechanics, the complete stress-strain relation, including linear elasticity, non-linear hardening, rapid stress drop and strain softening, is obtained. The rapids tress drop and strain softening are due to localizations of deformation and damage. The constitutive model, which analyzes the localizations of deformation and damage, is distinct from the conventional model, and theoretical predictions have shown consistency with the derived results.

\section{Introduction}

A project that NGI was involved in some years ago in southeast Asia has been chosen as an example to demonstrate the use in UDEC-BB. This work involved a triple lane traffic tunnel in volcanic tuff. Details of the actual jointed mass assumed to represent this tunnel are discribed later. The tensile stress is often produced in the unloading of the excavation of the slope and underground works. And the tensile stress increases with the increase of the size of tructure. Because of rock body is very small, so the deformation characteristics under the action of tensile stress does cannot ignore. It plays an important role in the stability evaluation of slope and underground. Under the action of tensile stress, the stress and strain of rock under uniaxial tension is important. Relationship has important theoretical and practical significance, in spite of the tensile stress on the rock. The deformation characteristics under the action of force are some valuable experimental research. But the theoretical research is not much, and the former model is only too low. In order to study the relationship between the ultimate load and the ultimate load, the main original results of the deformation and the actual observed deformation are obtained. For this reason, this paper focuses on the limit load of rock under uniaxial tension and later constitutive relation.

\section{Non Uniformity of Theoretical Model}

If the deformation of rock under uniaxial tensile loading is made up of the deformation of rock mass and microcrack, the deformation of rock under uniaxial tensile loading is composed of the linear elastic deformation of rock, patulousness of microcrack and the first stably patulous deformation 、 second unstably patulous deformation。

The relation of stress-strain of rock under uniaxial tensile loading takes the following form:

$$
\varepsilon_{i k}=\varepsilon_{i k}^{0}+\varepsilon_{i k}^{i 1}+\varepsilon_{i k}^{i 2}+\varepsilon_{i k}^{i 3}
$$


Where, $\varepsilon_{i k}^{0}, \varepsilon_{i k}^{i 1}, \varepsilon_{i k}^{i 2}, \varepsilon_{i k}^{i 3}$ respective is the linear elastic deformation of rock, patulous deformation of microcrack, the first stably patulous deformation of microcrack, and the second unstably patulous deformation of microcrack.

The linear elastic deformation of rock can be resolved by Hoke dinglv. It is assumed that the half of microcrack sizes randomly vary within the range $\left(c_{0}, c_{1}\right)$,

Where $c_{0}$ is the minimal half length, and the $c_{1}$ is the maximal half length. $\theta$ is the direction angle, $\sigma_{22}$ is the far field tensile stress. As shown in Fig 1.

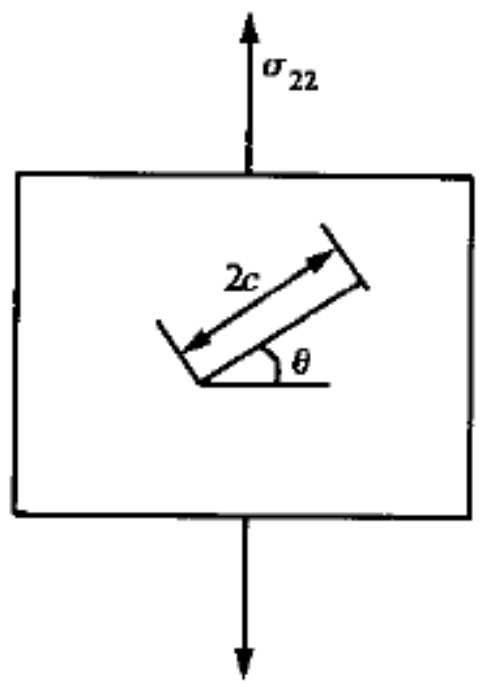

Fig. 1 Crack-weakened rock subjected to uniaxial tensile loading

Under uniaxial tensile loading, the stress intensity factors at the crack tip take the following form:

$$
\left\{\begin{array}{c}
K_{I}=\sigma_{22} \cos ^{2} \theta \sqrt{\pi c} \\
K_{\mathrm{II}}=\sigma_{22} \cos \theta \sin \theta \sqrt{\pi c}
\end{array}\right\}
$$

where $K_{\mathrm{I}}$ and $K_{\mathrm{II}}$ are the mode I and II stress intensity factors ,respectively .

The criterion of microcrack growth in a stable fashion is:

$$
K_{I}=K_{I C}
$$

in which $K_{I C}$ is the mode I critical stress intensity factor at weak plane. Substitution of (1) into (2), we have

$$
\sigma_{22}=\frac{K_{I C}}{\cos ^{2} \theta \sqrt{\pi c}}
$$

From (3), it is clear that the first microcrack to become unstable are oriented along $\theta=0$ direction and with maximum initial size $\mathrm{c}_{1}$, the corresponding threshold load is defined as:

$$
\sigma_{2 c}=\frac{K_{I C}}{\sqrt{\pi c}}
$$

Since microcrack sizes randomly vary within the range $\left(2 \mathrm{c}_{0}, 2 \mathrm{c}_{1}\right)$, the maximum and minimum 
microcrack size $c_{1}$ and $\mathrm{c}_{0}$ should be used in (3) instead of $\mathrm{c}$ in order to determine the critical domain of unstable microcrack growth.Therefore. (2) and (3) render the orientations $\left(0, \theta_{\mathrm{u} 1}\right)$ and $\left(0, \theta_{\mathrm{u} 0}\right)$ at which the largest and smallest microcraks become unstable and increase in size :

$$
\theta_{u 1}=\arccos \left(\frac{K_{1 c}}{\sigma_{2} \sqrt{\pi c_{1}}}\right)^{1 / 2}, \theta_{u 0}=\arccos \left(\frac{K_{1 c}}{\sigma_{2} \sqrt{\pi c_{0}}}\right)^{1 / 2}
$$

In addition, at a specified microcrack orientation $\theta$ within the range $\left(0, \theta_{\mathrm{u} 1}\right)$, the minimum microcrack size required to activate mode I growth can be evaluated from (3):

$$
c_{01}=\frac{K_{1 c}^{2}}{\sigma_{2}^{2} \pi \cos ^{4} \theta}
$$

If $0<\boldsymbol{\sigma}_{\mathbf{2}}<\boldsymbol{\sigma}_{\mathbf{2}}$, that is ,the stage of linear elasticity ,no microcrack growth begins . The compliance tensor attributable to a single open crack is given by (sumarac and krajcinovic 1987[8], J .W .Ju 1991[9])

$$
S_{i j}=\frac{2 \pi c^{2}\left(1-v_{0}^{2}\right)}{A_{0} E_{0}}\left(g_{2 i} g_{2 j}+g_{3 i} g_{3 j}\right)
$$

where $A_{0}$ is the representative element area of rock material, $E_{0}$ is Young's modulus , $v_{0}$ is poission 's ratio, $\boldsymbol{g}_{i j}$ are the components of the transformation matrix between the two coordinate system.

$$
[g]=\left[\begin{array}{ccc}
\cos ^{2} \theta & \sin ^{2} \theta & \sin 2 \theta \\
\sin ^{2} \theta & \cos ^{2} \theta & -\sin 2 \theta \\
-\frac{\sin 2 \theta}{2} & \frac{\sin 2 \theta}{2} & \cos 2 \theta
\end{array}\right]
$$

\section{The Complete Stress-strain Relation for Rocks}

The inelastic compliance tensor due to all preexisting open microcracks with the original sizes can be evaluated by:

$$
S_{i j}^{i 1}=\frac{\left(1-v_{0}^{2}\right)}{E_{0}} \rho \int_{0}^{\frac{\pi}{2}} \int_{c_{0}}^{c_{1}}\left(g_{2 i} g_{2 j}+g_{3 i} g_{3 j}\right) p(c) p(\theta) c^{2} d c d \theta
$$

where $\rho=2 \mathrm{~N} \pi / \mathrm{A}_{0}, \mathrm{~N}$ is the number of microcracks , $\mathrm{A}_{0}$ is the representative element area of rock material , $\mathrm{p}(\theta)$ and $\mathrm{p}(\mathrm{c})$ are the probability density function describing the distribution of orientations and sizes of microcracks in rock material ,respectively. $E_{0}$ is Young's modulus , $v_{0}$ is poission's ratio.

The overall effective compliance tensor can be obtained as

$$
S_{i j}=S_{i j}^{0}+S_{i j}^{i 1}
$$

in which $S_{i j}^{0}$ is the elastic and isotropic compliance of an undamaged rock material with Young's modulus $\mathrm{E}_{0}$ and poission 's ratiov. During the stage, the stress-strain relation take the following form :

$$
\varepsilon_{i j}=\left(S_{i j}^{0}+S_{i j}^{i 1}\right) \sigma_{2}
$$

If $\sigma_{2}=\sigma_{2 c}$, the microcracks in the plane $\theta=0$ with maximum initial size $2 \mathrm{c}_{1}$ become unstable and increase in size until reaching a characteristic length $2 \mathrm{c}_{2}$. 
If $\sigma_{2 c} \leq \sigma_{2}<\sigma_{2 c c}\left(\sigma_{2 c c}\right.$ is ultimate strength of rock material), that is ,the stage of pre-peak nonlinear hardening ,more microcracks become activated ,the compliance tensor contributions from stable and unstable microcracks $, S_{i j}^{i 1}, S_{i j}^{i 2}$ can be computed as follows, respectively:

$$
\begin{array}{r}
S_{i j}^{i 1}=\frac{\left(1-v_{0}^{2}\right)}{E_{0}} \rho \int_{\theta_{u 1}}^{\frac{\pi}{2}} \int_{c_{0}}^{c_{1}}\left(g_{2 i} g_{2 j}+g_{3 i} g_{3 j}\right) c^{2} p(c) p(\theta) d c d \theta \\
+\frac{\left(1-v_{0}^{2}\right)}{E_{0}} \rho \int_{\theta_{u 0}}^{\theta_{u 1}} \int_{c_{0}}^{c_{01}}\left(g_{2 i} g_{2 j}+g_{3 i} g_{3 j}\right) c^{2} p(c) p(\theta) d c d \theta \\
S_{i j}^{i 2}=\frac{\left(1-v_{0}^{2}\right)}{E_{0}} \rho \int_{0}^{\theta_{u 0}} \int_{c_{0}}^{c_{2}}\left(g_{2 i} g_{2 j}+g_{3 i} g_{3 j}\right) c^{2} p(c) p(\theta) d c d \theta \\
+\frac{\left(1-v_{0}^{2}\right)}{E_{0}} \rho \int_{\theta_{u 0}}^{\theta_{u 1}} \int_{c_{01}}^{c_{2}}\left(g_{2 i} g_{2 j}+g_{3 i} g_{3 j}\right) c^{2} p(c) p(\theta) d c d \theta
\end{array}
$$

where $\theta_{u 1}=\arccos \left(\frac{K_{1 c}}{\sigma_{2} \sqrt{\pi c_{1}}}\right)^{1 / 2}, \theta_{u 0}=\arccos \left(\frac{K_{1 c}}{\sigma_{2} \sqrt{\pi c_{0}}}\right)^{1 / 2}, c_{01}=\frac{K_{1 c}^{2}}{\sigma_{2}^{2} \pi \cos ^{4} \theta}$

The overall effective compliance tensor can be expressed as:

$$
S_{i j}=S_{i j}^{0}+S_{i j}^{i 1}+S_{i j}^{i 2}
$$

During the stage ,the stress-strain relation can be written as

$$
\varepsilon_{i j}=\left(S_{i j}^{0}+S_{i j}^{i 1}+S_{i j}^{i 2}\right) \sigma_{2}
$$

Under high stress, some microcrack arrested by the energy barriers, like grain boundaries, will satisfy the second growth criterion and propagate in an unstable fashion,causing localization of deformation and damage, the second growth criterion can be written as :

$$
K_{I}=K_{I C C}
$$

where $K_{\text {ICC }}$ is the critical value of SIF describing the resistance of rock material against microcrack growth.

According to (1)and (14), it is clear that the first microcrack to become unstable are oriented along $\theta=0$ direction and with maximum initial size $c_{2}$, the corresponding peak load is defined as :

$$
\sigma_{2 c c}=\frac{K_{I C C}}{\sqrt{\pi c_{2}}}
$$

If $\boldsymbol{\sigma}_{2}<\boldsymbol{\sigma}_{2 c c}$, no microcrack propagates in an unstable fashion .

If $\sigma_{2}=\sigma_{2 c c}$, that is ,the stage of rapid stress drop, some microcracks nearly normal to the tension direction and with maximum size $c_{2}$ propagate in an unstable fashion. As mentioned above, the distribution of sizes and orientations of microcracks in rock material can be described by the probability density function $\mathrm{p}(\mathrm{c})$ and $\mathrm{p}(\theta)$, respectively. If the number of microcracks normal to tensile direction is zero, it is assumed that microcracks whose orientations are within a small orientation scope $0 \leq \theta \leq \theta_{\mathrm{cc}}$ and with maximum initial size $\mathrm{c}_{2}$ propagate in an unstable fashion .

Once Eqn(15) is satisfied by microcracks whose orientations are within a small orientation scope $0 \leq \theta \leq \theta_{\mathrm{cc}}$ and with maximum initial size $\mathrm{c}_{2}$, they will experience the secondary unstable growth, which may cause a transition from the distributed damage to the localization of damage and a rapid stress 
drop at the transition strain $\varepsilon_{\mathrm{cc}}$.During the stage ,only microcracks whose orientations are within a small orientation scope $0 \leq \theta \leq \theta_{\mathrm{cc}}$ and with maximum size $\mathrm{c}_{2}$ propagate further and other microcracks undergo elastic unloading. In strain-controlled tests, the deformation which has received contributions from all microcracks during the first two stages concentrates gradually to the minority of microcracks experiencing the secondary growth, which results in a localization of deformation. Therefore, the macroscopic stress drop is the result of the localization of damage and deformation.

The relation between $c_{3}$ and $\sigma$ can be obtained approximately from the criterion (15), we have

$$
c_{3}=\frac{K_{I C C}^{2}}{\pi \sigma_{2}^{2}}
$$

The compliance tensor due to the microcracks experiencing the secondary growth can obtained by

$$
S_{i j}^{i 3}=\frac{\left(1-v_{0}^{2}\right)}{E_{0}} \rho \int_{0}^{\theta_{c c}} \int_{c_{2}}^{c_{3}} c^{2}\left(g_{2 i} g_{2 j}+g_{3 i} g_{3 j}\right) p(\theta) p(c) d c d \theta
$$

The compliance tensor contributions from unstable microcracks can be evaluated as:

$$
\begin{array}{r}
S_{i j}^{i 2}=\frac{\left(1-v_{0}^{2}\right)}{E_{0}} \rho \int_{\theta_{c c}}^{\theta_{u 2}} \int_{c_{0}}^{c_{2}}\left(g_{2 i} g_{2 j}+g_{3 i} g_{3 j}\right) c^{2} p(c) p(\theta) d c d \theta \\
+\frac{\left(1-v_{0}^{2}\right)}{E_{0}} \rho \int_{\theta_{u 2}}^{\theta_{u 3}} \int_{c_{01}}^{c_{2}}\left(g_{2 i} g_{2 j}+g_{3 i} g_{3 j}\right) c^{2} p(c) p(\theta) d c d \theta
\end{array}
$$

where $\theta_{u 3}=\arccos \left(\frac{K_{1 c}}{\sigma_{2 c c} \sqrt{\pi c_{1}}}\right)^{1 / 2}, \quad \theta_{u 2}=\arccos \left(\frac{K_{1 c}}{\sigma_{2 c c} \sqrt{\pi c_{0}}}\right)^{1 / 2}$

The compliance tensor attributing to stable microcracks can be computed as:

$$
\begin{aligned}
S_{i j}^{i 1} & =\frac{\left(1-v_{0}^{2}\right)}{E_{0}} \rho \int_{\theta_{u 3}}^{\frac{\pi}{2}} \int_{c_{0}}^{c_{1}}\left(g_{2 i} g_{2 j}+g_{3 i} g_{3 j}\right) c^{2} p(c) p(\theta) d c d \theta \\
& +\frac{\left(1-v_{0}^{2}\right)}{E_{0}} \rho \int_{\theta_{u 2}}^{\theta_{u 3}} \int_{c_{0}}^{c_{01}}\left(g_{2 i} g_{2 j}+g_{3 i} g_{3 j}\right) c^{2} p(c) p(\theta) d c d \theta
\end{aligned}
$$

During the stage ,the stress-strain relation can evaluated by:

$$
\varepsilon_{i j}=\left(S_{i j}^{0}+S_{i j}^{i 1}+S_{i j}^{i 2}+S_{i j}^{i 3}\right) \sigma_{2}
$$

During the stage of stress drop ,the strain maintains constant, we have

$$
\varepsilon_{2 c c}=\varepsilon_{2}
$$

where $\varepsilon_{2 c c}$ is the axial strain at peak loads $\sigma_{2 c c}, \varepsilon_{2}$ is the axial strain during stage of stress drop .

According to (20), the magnitude of the stress drop can be determined. It is assumed that the stage of rapid stress drop intersects that of tension softening at the point where the value of stress is $\sigma_{s c}$.

If $\sigma_{2}<\sigma_{s c}$,that is, the stage of strain softening. During the stage of strain softening, some of the microcracks which have undergone the secondary growth will propagate further, while other microcracks will simultaneously experience unloading. Meanwhile the growth criterion (15) must be satisfied by microcracks whose orientations are within a small orientation scope $0 \leq \theta \leq \theta_{\mathrm{cc}}$ and with maximum initial size $c_{2}$. The compliance tensor due to the microcracks experiencing the secondary growth ,unstable microcracks and stable microcracks $S_{i j}^{i 3}, S_{i j}^{i 2}, S_{i j}^{i 1}$ can be evaluated by 
(18),(19)and (20).

The stress-strain relation can be computed by

$$
\varepsilon_{i j}=\left(S_{i j}^{0}+S_{i j}^{i 1}+S_{i j}^{i 2}+S_{i j}^{i 3}\right) \sigma_{2}
$$

It is assumed that all microcrack are distributed uniformly in the orientations and sizes space The stress-strain relation for microcrack - weakened rock under uniaxial tensile loading can be expressed as (for plane strain):

$$
\varepsilon_{22}=\left\{\begin{array}{lc}
F_{0} \sigma_{2} & \left(0<\sigma_{2}\left\langle\sigma_{2 c}\right)\right. \\
{\left[F_{0}+F\left(\theta_{1}\right)\right] \sigma_{2}} & \left(\sigma _ { 2 c } \left\langle\sigma_{2}\left\langle\sigma_{2 c c}\right)\right.\right. \\
{\left[F_{0}+F\left(\theta_{2}\right)\right] \sigma_{2 c c}} & \left(\sigma _ { s c } \left\langle\sigma_{2}\left\langle\sigma_{2 c c}\right)\right.\right. \\
{\left[F_{0}+F\left(\theta_{2}\right)+F\left(\theta_{c c}\right)\right] \sigma_{2}} & \left(0<\sigma_{2}\left\langle\sigma_{s c}\right)\right.
\end{array}\right.
$$

For mesoscopic heterogeneous rock , the probability density function describing the distribution of the orientations of microcracks $\mathrm{p}(\theta)$ is approximated perfectly by Weibull distribution:

$$
p(\theta)=\frac{m}{\boldsymbol{\theta}_{0}}\left(\frac{\boldsymbol{\theta}}{\boldsymbol{\theta}_{0}}\right)^{m-1} \exp \left[-\left(\frac{\boldsymbol{\theta}}{\boldsymbol{\theta}_{0}}\right)^{m}\right]
$$

where $m$ is Weibull modulus, $\theta_{0}$ is characteristic angle 。

The probability density function describing the distribution of the sizes of microcracks $p(c)$ is approximated perfectly by Rayleigh function

$$
p(c)=A\left(\frac{c}{c_{00}}\right) \exp \left[-\left(\frac{c}{c_{00}}\right)^{2}\right]
$$

where $\mathrm{c}_{00}$ is characteristic length, $\mathrm{A}$ is the normalization constant $\left(\mathrm{A}=2 / \mathrm{c}_{00}\right)$

To simplify the analysis, it is assumed that $m=1$, the stress-strain relation for mesoscopic heterogeneous rock under uniaxial tensile loading is given as :

$$
\varepsilon_{22}=\left\{\begin{array}{lc}
F_{1} \sigma_{2} & \left(0<\sigma_{2}\left\langle\sigma_{2 c}\right)\right. \\
\left(F_{1}+F_{2}\right) \sigma_{2} & \left(\sigma _ { 2 c } \left\langle\sigma_{2}\left\langle\sigma_{2 c c}\right)\right.\right. \\
\left(F_{1}+F_{3}\right) \sigma_{2 c c} & \left(\sigma _ { s c } \left\langle\sigma_{2}\left\langle\sigma_{2 c c}\right)\right.\right. \\
\left(F_{1}+F_{3}+F_{4}\right) \sigma_{2} & \left(0<\sigma_{2}\left\langle\sigma_{s c}\right)\right.
\end{array}\right.
$$

\section{Conclusions}

In this paper, the single axial tension condition is studied by using the theory of micro mechanics, The paper gets some useful results as following:

(1) The main damage mechanism of uniaxial tension is self-similar, the deformation of rock in the tensile process can be decomposed into the deformation and crack of the rock matrix, the extension of the pattern, a stable expansion, and two times the instability of the extended deformation.

(2) The constitutive relation of micro cracks in the uniaxial tension process includes four steps: linear elasticity, nonlinear hardening, stress reduction and strain softening. The model has analyzed the mechanism of meso damage and constitutive relation of each stage. It is pointed out that the stress drop and strain softening are distributed damage to the injured. The unstable propagation of the micro crack is caused by the transition of the damage localization, and the damage localization is introduced into 
the material. The damage constitutive relation is another important difference between the previous damage models.

(3) The model can take into account the mesoscopic heterogeneity of rock material, As long as the fine distribution of the rock material, it can be concluded stress-strain relationship of whole process.

\section{Acknowledgement}

This work was financially supported by the Key research fund project: department of education of Sichuan province (No: 11ZA027).

\section{References}

[1] Nova R, Zaninetti A. Aninvestigation into the tensile behaviour of a schistose rock. International Journal Rock Mech. Sci.\& Geomech. Abstr., (1990),27:231 242

[2] Okubo S , Jin F, Akiyama M. Loading-rate dependency of uniaxial and indirect tensile strength. Journal of the Mining and Materials Processing Institute of Japan, (1993), 109:865 869

[3] Simo J C, Ju JW. Strain and stress -based continuum damage models. International Journal Solids Structures, (1987), 23:821 840

[4] Ortiz M. A constitutive theory for the inelastic behaviour of concrete. Mechanics of Materials, (1985), 4:67 93

[5] Budiansky B , O' Connell R J. Elastic moduli of a cracked solids. International Journal Solids Structure,(1976), 12: 81 79

[6] Sumarac D, Krajcinovic D. A selfconsistent model for microc-rack-weakened solids. Mechanics of Materials, (1987), 6:39 52

[7] Ju J W. On two-dimensional selfconsistent micro mechanical damage models for brittle solids. International Journal of Solids and Structures, (1991), 27:227 258

[8] Oda, M. Similarity rule of crack geometry in statistically homogeneous rock masses. Mech. Mater. (1984), 3: 119-129

[9] Shao, J.F., Hoxha, D., Bart, M., 1999. Modelling of induced anisotropic damage in granites. Int. J. Rock Mech. Min. Sci. 36, 1001-1012 